\title{
VIAS DE SÍNTESE LINEAR E CONVERGENTE - QUAL É MAIS VERDE?
}

\author{
Adélio A. S. C. Machado* \\ Departamento de Química, Faculdade de Ciências, Universidade do Porto, R. Campo Alegre, 687, 4169-007 Porto, Portugal
}

Recebido em 9/1/11; aceito em 18/3/11; publicado na web em 10/6/11

\begin{abstract}
LINEAR VS CONVERGENT SYNTHETIC PATHWAY - WHICH IS GREENER? A comparative study of a convergent and the linear synthetic pathway with respect to their relative greenish allowed the quantification of the advantages of the former with respect to atomic productivity as well as robustness. The calculations show that convergent pathways provide a decrease of costs together with a decrease of $\mathrm{E}$ factor and an increase of atomic economy which means that greenish is accompanied by an economic advantage. The influence of other features of the convergent pathways synthesis on the improvement of the synthesis greenish is discussed qualitatively.
\end{abstract}

Keywords: Green Chemistry; linear pathway; convergent pathway.

\section{INTRODUÇÃO}

Num artigo anterior ${ }^{1}$ discutiu-se como a logística da via de síntese influencia as suas caraterísticas de verdura, pelo que deve ser considerada no respetivo design no âmbito da Química Verde (QV). O objetivo fundamental deste artigo é realizar uma comparação das vias de síntese convergente e linear neste contexto, mais precisamente quanto à sua adequabilidade relativa para proporcionar verdura. Embora a literatura da QV refira, ocasionalmente, que as vias convergentes têm vantagens sobre as lineares quanto à poupança de recursos materiais, ${ }^{2}$ tanto quanto o autor sabe, nunca foi realizado nenhum estudo sobre as relações quantitativas entre a convergência da síntese e a respetiva verdura. Em contraste, as vantagens logísticas das vias convergentes têm sido bastante estudadas no âmbito do escalamento das sínteses do laboratório para a indústria feito no desenvolvimento de processo, ${ }^{3-5}$ em particular quanto à minimização de resíduos e de custos; ${ }^{5}$ e mesmo, embora mais raramente, em química laboratorial, focando em especial a vantagem das vias convergentes para maximizar o rendimento global. ${ }^{6,7}$

$\mathrm{O}$ artigo começa por uma discussão sobre as dificuldades em estabelecer critérios de comparação de vias de síntese alternativas, um aspeto básico para a escolha da melhor via, a que não se dá muita atenção no ensino universitário da Química e que facilitará a compreensão do que se segue.

O trabalho foi realizado seguindo a metodologia descrita na ref. 1: com base em raciocínios sobre a logística de reagentes e grupos de átomos em modelos simples de vias de síntese convergente e linear, esta última usada como base de comparação, fizeram-se cálculos de custos e de métricas de massa de QV, para investigar a interrelação destas grandezas quando se altera a natureza da via e a sua logística, e como se pode aumentar a verdura atómica da via de síntese por otimização desta. A verdura atómica é aferida pela produtividade atómica, ${ }^{8}$ que é medida pelas métricas de massa economia atómica e fator $\mathrm{E},{ }^{9-11} \mathrm{e}$ aumenta com o bom cumprimento dos dois primeiros dos Doze Princípios da Química Verde (Prevenção de resíduos e Economia atómica), ${ }^{12-14}$ que induz a utilização proveitosa dos átomos em reação. Mais detalhadamente, o trabalho para visualizar as relações quantitativas entre as opções tomadas no design da via convergente

*e-mail: amachado@fc.up.pt e a verdura obtida consistiu em comparar um modelo de via convergente com uma via linear com o mesmo número de passos e avaliar os efeitos da ocorrência na via de um passo de baixo rendimento na respetiva robustez em função do seu posicionamento ao longo da via.

Numa última secção, discutem-se os efeitos de outros fatores caraterísticos das vias de síntese na respetiva verdura relativa, embora não se quantifiquem as comparações.

\section{AS DIFICULDADES DA COMPARAÇÃO DE VIAS DE SÍNTESE ALTERNATIVAS}

Na atividade de otimização de uma via de síntese para a sua utilização industrial na manufatura de um composto, os químicos de processo têm frequentemente necessidade de comparar vias de síntese alternativas, com vista a escolher a de menor complexidade - que, em princípio, permitirá obter maior eficácia no fabrico. No entanto, como qualquer comparação de sistemas complexos, seja qual for o seu tipo, esta tarefa quase nunca é simples - e nem sempre permite chegar a um resultado inequívoco. Esta seção do artigo tem como objetivo alertar os químicos de formação académica, com atividade estritamente laboratorial, para as dificuldades de aferir a complexidade das vias de síntese, mesmo que apenas em termos relativos, quando elas são constituídas por uma série numerosa de reações químicas e, muito particularmente, quando têm estruturas diferentes.

\section{Avaliação académica da complexidade das vias de síntese}

Os químicos académicos avaliam frequentemente a complexidade das vias de síntese linear que inventam por meio de dois critérios (cujos resultados são vulgarmente designados por métricas): o número de etapas que usaram na síntese $(n)$ e o rendimento global, obtido por multiplicação dos rendimentos das sucessivas etapas, usando, normalmente (e otimisticamente!), o melhor rendimento que obtiveram em réplicas de cada uma das etapas da síntese linear:

$$
\mathrm{R}_{\text {Global }}=\Pi_{n} \mathrm{R}_{\mathrm{i}} \quad \mathrm{i}=1, \ldots, n
$$

Estes critérios são de fácil compreensão e cálculo e servem razoavelmente as necessidades dos químicos laboratoriais porque, 
na sua atividade académica, em geral, o problema de comparar a complexidade de vias de síntese alternativas não se põe com acuidade - normalmente, as comparações feitas são publicadas nos artigos em que os químicos relatam as novas sínteses, para fazer ressaltar as caraterísticas superiores destas relativamente às descritas anteriormente (nomeadamente, serem constituídas por menos etapas e realizadas com maior rendimento), geralmente com um certo grau de autocomplacência. ${ }^{6}$ As comparações não têm quaisquer consequências económicas (são meramente académicas!), pelo que quase nunca se escrutina a sua precisão.

\section{Avaliação da complexidade das vias de síntese na I\&D industrial}

A situação com que se deparam os químicos de processo, cuja tarefa é conceber ou otimizar vias de síntese para usar à escala industrial, é completamente diferente. O seu objetivo é fabricar o composto com custos mínimos - e como qualquer pequena simplificação da via pode ser importante para a redução de custos, a comparação de rotas alternativas para obter o composto exige precisão.

Neste contexto, é importante salientar que os critérios anteriores podem ser mais ou menos ambíguos em consequência de várias limitações, pelo que não podem ser empregues cegamente. Como a comparação de vias tem consequências económicas para o processo industrial, impõe-se discutir estas limitações, que são listadas no Quadro 1 e analisadas brevemente a seguir.

Quadro 1. Dificuldades na caraterização da complexidade das vias de síntese

\begin{tabular}{|c|}
\hline Número de etapas \\
Definição de etapa \\
Definição do(s) reagente(s) inicial(ais) \\
Contagem de etapas \\
Rendimento global \\
Cálculo para sínteses convergentes \\
\hline
\end{tabular}

\section{Número de etapas}

Quanto a este critério, os problemas surgem não só nas definições de etapa e de reagente inicial, o que tem implicações na contagem das etapas, mas também, e mais criticamente, no fato de o número de etapas não ser um bom critério de avaliação de complexidade quando a via de síntese é convergente.

\section{Definição de etapa}

O conceito de etapa usado na literatura química é algo vago, já que o termo é empregue com significados diferentes pelos químicos laboratoriais; por exemplo, uma etapa pode ser, conforme os autores:

(i) um fenómeno de rutura/formação de ligações - reação química ou, segundo a nomenclatura seguida mais à frente neste artigo, ${ }^{1}$ passo químico;

(ii) a resolução de uma mistura racémica: isolamento de um dos enatiómeros por precipitação de um sal diastereoisomérico - é uma reação química, mas é usada para realizar uma separação (designado por passo de separação);

(iii) qualquer outro processo ou operação usados na via, em geral representada por uma seta no respetivo diagrama esquemático.

Em consequência, a contagem de etapas e passos (a diferença entre passo e etapa foi descrita na ref. 1) não é precisa, por exemplo, a separação em (ii) pode ser, ou não, contada como uma unidade.

Para os químicos de processo, o conceito de etapa é definido por um critério pragmático: é o conjunto de processos que termina com o isolamento de um composto intermediário ou do composto final. O número de etapas é, portanto, o número de compostos isolados ao longo da via de síntese. Quando o químico de processo, ao otimizar a síntese, elimina uma separação (realiza a chamada telescopagem), reduz o número de etapas de uma unidade - com o fito de diminuir a complexidade da síntese e minimizar os seus custos, por exemplo, quanto a tempo da execução fabril, ao uso de solventes, ao dispêndio de energia nas operações unitárias usadas para a separação (destilação, cristalização, filtração, secagem, etc.). Esta prática, porém, pode não melhorar o rendimento global e pode afetar a qualidade da via de síntese, se provocar contaminação do intermediário isolado por impurezas que tenham consequências na qualidade do produto final. Este facto mostra que nem sempre a diminuição do número de etapas corresponde a uma simplificação aceitável da síntese - e que, portanto, o uso do número de etapas como critério de comparação da simplicidade e eficiência de vias de síntese pode ser problemático.

Em termos práticos, o químico de processo, antes de realizar uma telescopagem, tem de analisar e compreender muito bem os passos que podem ser agregados para se assegurar que a sua aglomeração não é inconveniente..$^{15} \mathrm{Um}$ aspeto importante a considerar nesta análise, além da necessidade de realizar o isolamento de que se gostaria de prescindir com o fim de se eliminar impurezas críticas, é a adequabilidade/inadequabilidade do respetivo intermediário para ser isolado. Esta caraterística é complexa: depende, por exemplo, das propriedades físicas, da estabilidade química, da toxicidade/potência biológica do composto (tendo em vista a proteção ocupacional dos operadores), da facilidade de isolamento (cristalização), etc. - em suma, na prática, deve-se avaliar a complexidade do isolamento cotejando-a com sua eficácia para eliminar impurezas indesejáveis. A telescopagem pode ser uma boa solução em certos casos - mas inadequada em outros.

Em conclusão, o número de etapas não é um critério absoluto para a comparação de vias sintéticas.

\section{Definição do(s) reagente(s) inicial(ais)}

Esta definição é importante para se saber onde se deve começar a contar as etapas, mas também a este respeito há uma margem de indefinição, porque o "zero" pode ser definido de vários modos, por exemplo: um composto disponível comercialmente; um produto natural a partir do qual se pode realizar a síntese; um composto cuja síntese está bem descrita na literatura, mas que não está no mercado comercial (a sua obtenção exige etapas prévias, que podem ou não ser contadas); um composto não disponível comercialmente, mas cuja síntese pode ser encomendada a uma empresa exterior, especializada em sínteses por encomenda (custom synthesis); na indústria farmacêutica há ainda um outro aspeto que pode condicionar a escolha dos reagentes iniciais: a regulamentação de fabrico quanto à natureza das matérias-primas pode exigir reagentes de partida ou intermediários fabricados segundo normas estritas, definidas em cada caso.

Em química académica, a escolha dos reagentes de partida é ditada quase sempre pela facilidade de acesso e aprovisionamento dos mesmos, embora os custos possam também pesar, bem como o próprio objetivo do trabalho a realizar (por exemplo, este pode ser sintetizar um composto a partir de um determinado produto natural). Pelo contrário, o químico de processo tem de abordar o problema com outro alcance, numa perspetiva dinâmica e num prazo dilatado no tempo. Um dos fatores que deve avaliar é, por exemplo, o fornecimento de matérias-primas quanto à estabilidade de fornecimento e de preços, com vista a obter uma via sintética que se possa manter em operação ao longo do tempo, sem vicissitudes de aprovisionamento dos reagentes - por exemplo, na prática, deve privilegiar matériasprimas disponibilizadas no mercado por vários fabricantes, para melhor garantir a sua aquisição a longo prazo (no caso da Química Orgânica Fina, que exige reagentes elaborados, esta condição nem sempre pode ser cumprida, sendo então necessário enveredar por 
soluções de outro tipo, por exemplo, contratos de fornecimento plurianuais com empresas de síntese por encomenda).

\section{Contagem de etapas}

Este terceiro aspeto, que diz respeito ao uso do número de etapas como medida da complexidade da síntese, é ainda mais problemático que os anteriores - porque envolve a própria natureza conceptual da síntese (linear ou convergente). A comparação do número de etapas entre duas vias de síntese só é lícita quando a sua estrutura for a mesma - se uma for linear e a outra convergente, ou se forem ambas deste último tipo, mas com diferentes formas e graus de convergência, o número de etapas não é uma medida objetiva da complexidade e da eficiência de cada uma delas. Um exemplo ilustrará esta afirmação.

$\mathrm{Na}$ Figura 1 apresentam-se os esquemas de duas vias de síntese com o mesmo número de etapas (10), uma linear, outra convergente e com dois ramos aproximadamente equilibrados. Os números de etapas e de ligações formadas no produto são os mesmos nas duas vias, embora a sequência do estabelecimento das ligações seja diferente. Quando se comparam de modo intuitivo as duas vias quanto a complexidade, os resultados são subjetivos: por um lado, pode-se dizer que a complexidade da via convergente é menor do que a da linear, porque o ramo mais longo da primeira (a partir de A, sequência $1 \rightarrow 3 \rightarrow 4 \rightarrow 5 \rightarrow 10$ ) envolve 5 etapas, ao passo que a linear envolve 10 - em face destes números, pode-se até quantificar a comparação e dizer que a eficácia da síntese convergente é o dobro da linear; por outro lado, também se pode contra-argumentar que a complexidade das duas vias é igual, porque ambas envolvem globalmente 10 etapas. Assim, quando a estrutura não é a mesma, contar as etapas para aferir a simplicidade da via de síntese e avaliar a sua eficácia é problemático.

\section{VIAS DE SÍNTESE}

\section{(1) VIA LINEAR}

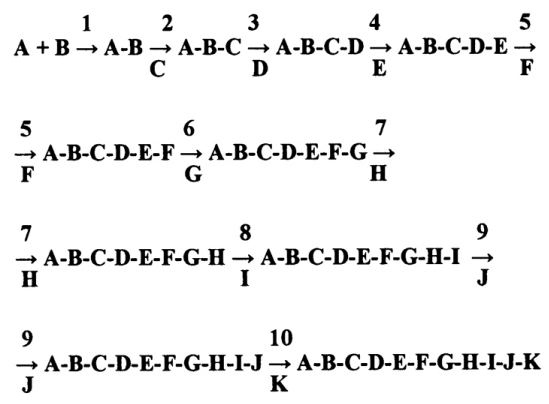

\section{(2) VIA CONVERGENTE}

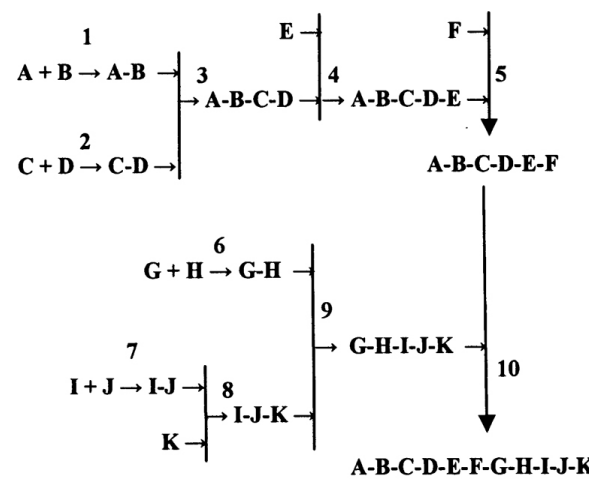

Figura 1. Vias de síntese (1) linear e (2) convergente com o mesmo número de passos
Em termos práticos, o número de etapas deve ser usado apenas como uma medida preliminar, parcial, da complexidade da via, a ser avaliada conjuntamente com outras caraterísticas desta, por exemplo, as propriedades dos intermediários, tais como a estabilidade e a facilidade de cristalização, o potencial para a telescopagem, etc. - enfim, todas as caraterísticas que definem a natureza das etapas a concretizar, não só quanto às reações químicas como também quanto às operações unitárias requeridas para isolar os compostos.

\section{Rendimento global}

A aplicação do segundo critério acima, o cálculo do rendimento global (Equação 1), para fazer as comparações, envolve frequentemente ainda mais problemas que a contagem do número de etapas - e, no caso das sínteses convergentes, pode até conduzir a resultados ambíguos. Tal como no caso da contagem das etapas, o rendimento global depende da definição da etapa inicial - mas como para as sínteses convergentes há necessariamente diversos pontos de partida, o cálculo se complica.

Os problemas surgidos podem ser ilustrados com as vias na Figura 1. Admitindo, irrealistamente mas para simplificar, que todas etapas (1 a 10) têm o mesmo rendimento, por exemplo, $90 \%$, o rendimento global da via linear será 35\% $\left(=0,9^{10}\right)$. Quando se usa a Equação 1 para calcular o rendimento da via convergente, o resultado varia com a linha escolhida: se se segue a linha com mais etapas (a partir de A, $1 \rightarrow 3 \rightarrow 4 \rightarrow 5 \rightarrow 10$, cinco etapas), o rendimento global será de $59 \%\left(=0,9^{5}\right.$ ), mas para a linha de baixo (a partir de $\mathrm{K}, 8 \rightarrow 9 \rightarrow 10$, três etapas), obtém-se o valor $73 \%\left(=0,9^{3}\right)$. Qualquer um destes dois rendimentos calculados para a síntese convergente é superior ao da via de síntese linear.

O procedimento anterior de usar a linha "mais longa" (com mais etapas) para obter o rendimento de uma síntese convergente é habitual, mas não tem significado químico absoluto - sendo por isso inadequado. Por exemplo, se se admitir que as etapas 8 e 9 têm o rendimento de $70 \%$ e as outras o já usado valor de $90 \%$, o rendimento global calculado para a linha inferior $(8 \rightarrow 9 \rightarrow 10)$, só com 3 etapas, será de $44 \%\left(=0,7^{2} \times 0,9\right)$, inferior ao calculado acima para a via com cinco etapas (59\%), pelo que deve prevalecer sobre este último, já que limita mais o processo global. Neste caso, tal como os anteriores, o valor obtido é superior ao da via linear. Esta superioridade quanto ao rendimento global, encontrada frequentemente (mas não garantida em absoluto, ver adiante), é uma vantagem das vias convergente sobre as lineares.

Por outro lado, o cálculo do rendimento pode envolver outras variáveis que não apenas os rendimentos das etapas individuais. Por exemplo, se a síntese consistir em adicionar sucessivamente ao fragmento A (uma molécula volumosa), os outros grupos funcionais $(\mathrm{B}, \ldots, \mathrm{K}), \mathrm{A}$ deve ser designado como o reagente de partida, porque é ele que contribui com maior massa para o produto - neste caso, o cálculo do rendimento deve ser feito segundo a linha $(1 \rightarrow 3 \rightarrow 4 \rightarrow 5 \rightarrow 10)$. Outro exemplo: se o reagente I for muito dispendioso, o rendimento deve ser calculado segundo a linha $(7 \rightarrow 8 \rightarrow 9 \rightarrow 10)$, porque é a sua utilização que define a economia global da via de síntese (lateralmente, note-se que neste caso a via linear pode ser mais conveniente do que a convergente, porque usa o reagente dispendioso só no fim - não havendo o problema de o manter incólume ao longo da via).

Por outro lado, como normalmente os rendimentos das etapas são diferentes, as quantidades de reagentes de partida das diversas linhas devem ser ajustadas para se obter as proporções adequadas de reagentes intermediários nos pontos de convergência. Por exemplo, sendo $\mathrm{R}_{1} \mathrm{e}$ $R_{2}$ os rendimentos das etapas 1 e 2, para se realizar a etapa 3, as quantidades obtidas de $\mathrm{AB}$ e $\mathrm{CD}$ devem ser ajustadas mediante a realização das etapas 1 e 2 com quantidades de reagentes na proporção $1:\left(R_{1} / R_{2}\right)$. 
Além disto, quando se pretende comparar duas vias convergentes, por exemplo, uma com convergência perto da partida e outra perto do produto final, a situação pode ser muito mais complicada que a do caso da Figura1. Também, os resultados de cálculos pela Equação 1 variam muito com os valores dos rendimentos individuais, porque envolvem muitos fatores ou potências cujos expoentes são frequentemente elevados. Este facto é ilustrado pela Tabela 1 (respigada de uma tabela mais completa da ref. 7), que mostra (nas linhas) como o rendimento global varia com o rendimento das etapas $\left(\mathrm{R}_{\mathrm{i}}\right.$ na Equação 1); bem como (nas colunas) o rendimento é rapidamente erodido quando o número de etapas $n$ aumenta muito (para sentir esta influência, pode-se também repetir os cálculos descritos atrás no texto com outros valores de rendimento, por exemplo, 80 e $60 \%$ em vez de 90 e $70 \%$, respetivamente).

Tabela 1. Rendimento global (\%) de uma via de síntese em função do número de etapas e dos rendimentos das etapas individuais (supostos todos iguais)

\begin{tabular}{ccccc}
\hline \multirow{2}{*}{\begin{tabular}{c}
$\mathrm{N}^{\text {de }}(n)$ \\
\cline { 2 - 5 }
\end{tabular}} & $50 \%$ & $70 \%$ & $75 \%$ & $90 \%$ \\
\hline 1 & 50 & 70 & 75 & 80 \\
5 & 3,1 & 17 & 24 & 59 \\
10 & 0,09 & 3 & 5,6 & 35 \\
15 & 0,003 & 0,5 & 1,3 & 20,6 \\
20 & 0,0001 & 0,08 & 0,28 & 12,2 \\
25 & 0,0000025 & 0,02 & 0,07 & 7,2 \\
\hline
\end{tabular}

Em suma, a validade do cálculo, vulgarmente feito, do rendimento global de uma via convergente, com base na identificação da sequência de etapas mais longa e aplicação da Equação 1 a essa linha, é problemática: frequentemente, o rendimento global tem de ter em atenção outros fatores que não apenas os rendimentos individuais das reações realizadas nessa sequência. Consequentemente, os valores obtidos não podem ser usados como base de um critério de eficácia das vias de síntese convergentes - nem permitem aferir com precisão a complexidade relativa de vias alternativas para obter um mesmo produto.

\section{Conclusões}

Para o químico de processo, o número de etapas e o rendimento global são apenas dois dos muitos critérios pelos quais uma via de síntese deve ser avaliada - e o rendimento global é um valor que pode resultar de um cálculo equívoco, sem significado químico preciso e cuja utilização como métrica pode ter consequências práticas nefastas. Como se discutirá adiante, as vias de síntese convergente, quando comparadas com as lineares, têm caraterísticas muito atraentes, quer para trabalho de laboratório quer, principalmente, para o fabrico industrial, nomeadamente quanto ao aumento da verdura conseguida, mas essas vantagens não incluem necessariamente um maior rendimento global.

As vias de síntese convergentes são sistemas complexos de processamento de átomos, que têm de ser abordadas com visão holística - as reações que as integram têm de ser consideradas como um todo, o que não é feito quando se identifica a linha de etapas sequenciais mais longa para calcular o rendimento global e se ignora o impacto das outras (uma atitude reducionista que só por acaso feliz não conduz a resultados errados!). Esta situação ilustra a natureza sistémica da química, ${ }^{16}$ que os químicos académicos, geralmente formados no quadro racionalista da ciência, têm frequentemente dificuldade em aceitar.

\section{CÁLCULOS}

Admitiu-se, como na ref. 1, que: as vias de síntese eram constituídas por 10 passos de reações de adição; após cada passo, o produto obtido era isolado dos reagentes não reagidos e dissolvido no solvente para realizar o passo seguinte; e as proporções de reagentes usados eram sempre as estequiométricas. Os valores numéricos usados para definir os modelos foram também os usados na ref. 1. Nestas condições, é fácil realizar cálculos de custos (e das respetivas percentagens de aumento) de reagentes, de solventes e de totais, bem como da economia atómica e do fator E, para os dois tipos de vias, sendo a via linear usada como base de comparação.

Para se realizar uma comparação quantitativa das vias de síntese convergente relativamente à linear (Exemplo 1) realizaram-se cálculos da variação de custos e de parâmetros de produtividade atómica (economia atómica e factor E) quando se passava da via linear para a via convergente apresentada na Figura 1. Para a via de síntese linear os cálculos foram realizados na ref. 1 . Os cálculos para a via convergente são mais elaborados, porque se tem de atender ao facto de as quantidades estequiométricas de reagentes a usar variarem com o número de passos de cada uma das sub-linhas conducentes ao produto final. Começou-se por calcular essas quantidades (Tabela 2, em que as proporções se referem ao rendimento de 90\%) e, depois, fizeram-se os cálculos dos custos e dos parâmetros de produtividade atómica a partir destes valores. Os cálculos foram realizados num Excel semelhante ao usado na ref. 1, mas que incluía à partida o cálculo das quantidades de reagentes com base na Tabela 2. O Excel pode ser obtido do autor por meio de pedido feito por e-mail.

Tabela 2. Quantidades de reagentes usados na síntese convergente

\begin{tabular}{|c|c|c|c|c|c|c|}
\hline $\begin{array}{l}\mathrm{N}^{\mathrm{o}} \text { de } \\
\text { passos }(n)\end{array}$ & 0 & 1 & 2 & 3 & 4 & 5 \\
\hline \multirow[t]{15}{*}{ Proporção* } & 1,00 & 0,90 & 0,81 & 0,73 & 0,66 & 0,59 \\
\hline & \multicolumn{6}{|c|}{ Reagentes e reações para obter o produto } \\
\hline & $\mathbf{A}+\mathbf{B} \rightarrow$ & $\mathrm{AB}$ & & & & \\
\hline & & + & & & & \\
\hline & $\mathbf{C}+\mathrm{D} \rightarrow$ & $\mathrm{CD}$ & $\rightarrow \mathrm{ABCD}$ & & & \\
\hline & & & + & & & \\
\hline & & & $\mathbf{E}$ & $\rightarrow \mathrm{ABCDE}$ & & \\
\hline & & & & + & & \\
\hline & & & & $\mathbf{F}$ & $\rightarrow \mathrm{ABCDEF}$ & \\
\hline & & & & & + & \\
\hline & & & $\mathbf{G}+\mathbf{H} \rightarrow$ & $\mathrm{GH}$ & $\rightarrow$ GHIJK & $\rightarrow \mathrm{AB} \ldots \mathrm{JK}$ \\
\hline & & & & + & & \\
\hline & & $\mathbf{I}+\mathbf{J}$ & $\rightarrow \mathrm{IJ}$ & $\rightarrow \mathrm{IJK}$ & & \\
\hline & & & + & & & \\
\hline & & & $\mathbf{K}$ & & & \\
\hline
\end{tabular}

(*) Proporção = Rendimento ${ }^{n}$ (os valores referem-se ao rendimento de 90\%)

Para se analisar a robustez da via convergente (Exemplo 2), considerou-se a via de síntese convergente com 10 passos de rendimento individual $90 \%$, admitiu-se que o rendimento de um dos passos baixava para metade (45\%) e deslocou-se sucessivamente este passo ao longo da síntese, da posição 1 até à posição 10 . Os resultados obtidos foram comparados com os obtidos por cálculos referentes à mesma situação para a via linear, apresentados na ref. 1 .

\section{RESULTADOS E DISCUSSÃO}

Embora a análise das vantagens e desvantagens das vias de sínteses lineares e convergentes seja uma tarefa sistémica, no caso 
simplista aqui usado pode realizar-se uma abordagem reducionista da sua comparação, para evidenciar que as vias de síntese convergentes apresentam muitas vantagens sobre as lineares quando se procura obter verdura. As vantagens mais gerais das vias de síntese convergentes sobre as lineares são listadas no Quadro 2 e discutidas a seguir.

Quadro 2. Comparação das vias linear e convergente

$$
\begin{aligned}
& \text { Velocidade de produção e custos } \\
& \text { Robustez } \\
& \text { Menos trechos de via improdutivos } \\
& \text { Controlo de impurezas } \\
& \text { Aquisição exterior de intermediários }
\end{aligned}
$$

\section{Custos e verdura}

Uma síntese convergente permite realizar o processamento paralelo dos diversos ramos simultaneamente, pelo que a duração do ciclo de fabrico diminui - e a velocidade de produção aumenta. Este facto resulta de a massa total de materiais intermediários a processar ser menor, o que implica, por exemplo, usar menores volumes de solventes e despender menos tempo a realizar as operações. Em consequência disto, o uso de vias convergentes faz diminuir os custos de manufatura, quer os de matérias-primas e reagentes, quer os de conversão.

Para ilustrar quantitativamente esta situação, bem como os efeitos quanto a métricas de produtividade atómica, no Exemplo 1 comparase as vias convergente e linear da Figura 1.

\section{Exemplo 1}

Na Figura 2 apresenta-se a variação de custos quando a síntese é realizada pela via convergente, relativamente à linear, em função do rendimento dos passos individuais. Quando este rendimento for de $90 \%$, os custos de reagentes, solventes e totais na via convergente são reduzidos de 23,47 e $31 \%$, respetivamente, com respeito à linear. A rendimentos mais baixos, ocorre uma redução exponencial dos custos com o rendimento. Mesmo no caso ideal de o rendimento dos passos individuais ser $100 \%$, em que os custos de reagentes se mantêm, ocorre uma redução de $35 \%$ no custo de solventes (que mede grosseiramente o custo de fabrico ${ }^{1}$ ), que se traduz num abaixamento dos custos totais de $13 \%$. Este comportamento resulta de a síntese convergente ter de processar menores quantidades de compostos intermediários.

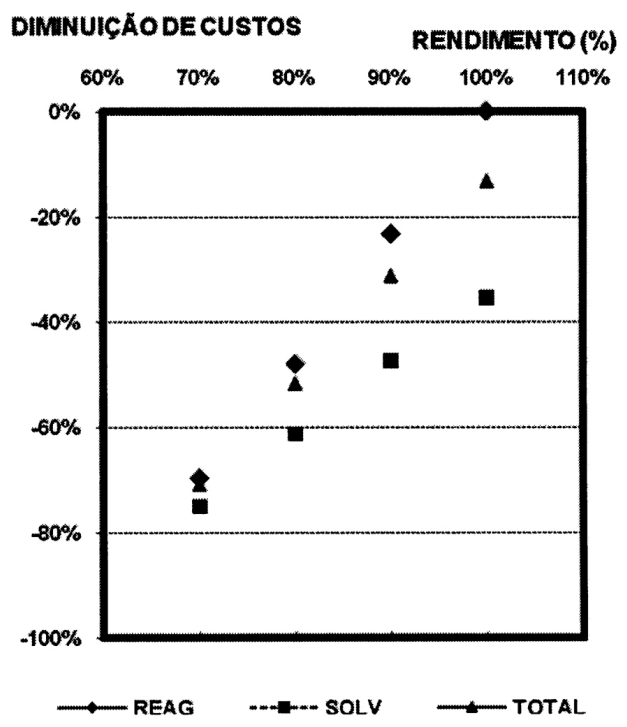

Figura 2. Diminuição de custos da via de síntese convergente relativamente à linear em função do rendimento individual dos passos
Na Figura 3 comparam-se os valores do fator E e da economia atómica para as duas vias, em função do rendimento dos passos individuais. A figura mostra que o fator E é sempre maior para a via linear e a economia atómica para a via convergente - e tanto mais quanto o rendimento for mais baixo. Estes resultados evidenciam que a produtividade atómica da via de síntese convergente é maior que a da linear. Assim, a comparação mostra que o abaixamento de custos na via de síntese convergente é acompanhado por obtenção de maior verdura, tal como sucedeu na análise sobre vias lineares. ${ }^{1}$
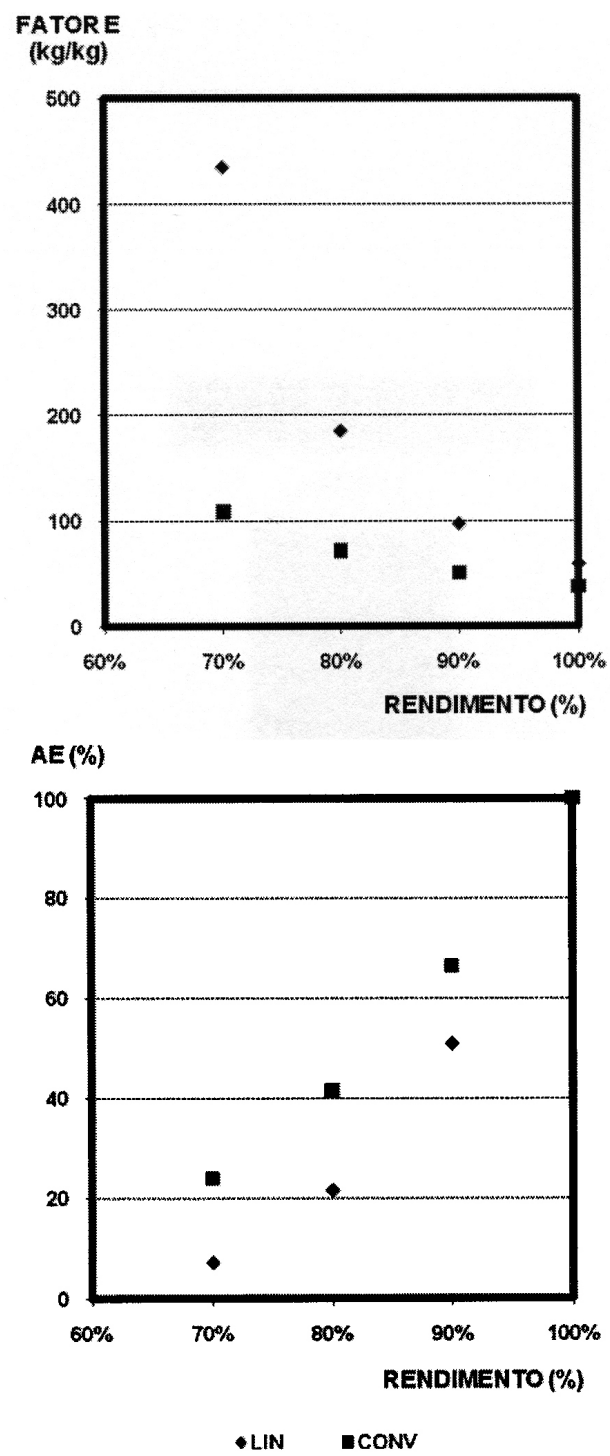

Figura 3. Comparação das métricas de produtividade atómica para as vias de síntese convergente e linear em função do rendimento individual dos passos. Em cima: fator E. Em baixo: economia atómica

\section{Robustez}

As vias de síntese convergentes são menos afetadas que as lineares por flutuações de rendimento dos passos, diluições, custos de reagentes, etc., porque a sua estrutura ramificada dispersa os efeitos pelos diversos ramos de forma menos igualitária - as alterações provocadas propagam-se em menor extensão e ficam mais localizadas no ramo em que ocorrem. Por isso, as vias convergentes são mais robustas que as lineares - resistem melhor a variações aleatórias nas condições de realização. O Exemplo 2 ilustra quantitativamente este facto. 


\section{Exemplo 2}

Neste exemplo, considera-se a via de síntese convergente com 10 passos de rendimento individual $90 \%$, admite-se que o rendimento de um dos passos baixa para metade (45\%) e desloca-se sucessivamente este passo ao longo da síntese, da posição 1 até à posição 10 .

A Figura 4 resume os resultados obtidos quanto a variações de custos de reagentes, de solventes e totais, com o deslocamento do passo de baixo rendimento. A comparação desta figura com a Figura 2 da ref. 1 , referente à via linear, mostra que os aumentos de custos para o presente caso da via convergente são sempre menores do que para a linear - na maioria dos casos, as diferenças são substanciais. Por exemplo, no caso mais desfavorável, quando o passo de baixo rendimento é o último, o aumento de custos para a via convergente é de apenas $80 \%$, ao passo que para a linear é de $100 \%$. Note-se que o rendimento global é sempre o mesmo em cada uma das vias, embora superior para a via convergente (ver acima), devido ao menor número de passos em linha (máximo de cinco na linha $1 \rightarrow 2 \rightarrow 3 \rightarrow 4 \rightarrow 5$ ).

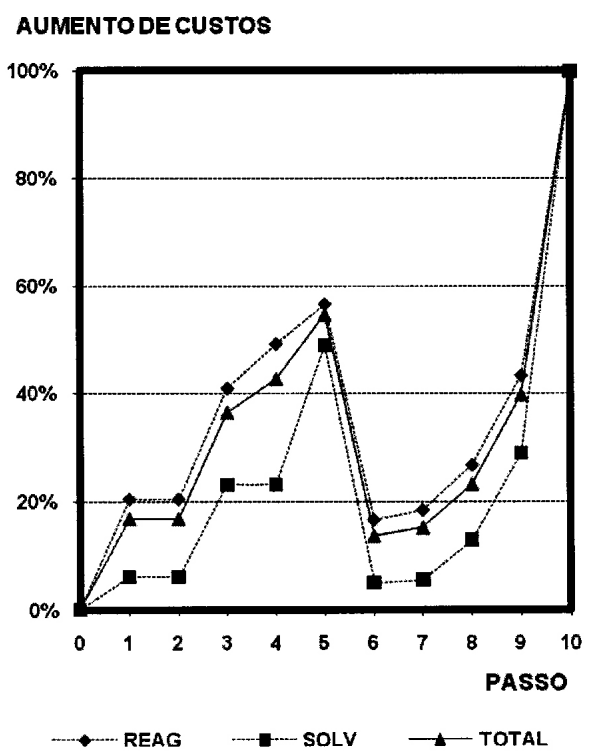

Figura 4. Aumento de custos em função da posição do passo com rendimento $45 \%$ na via de síntese convergente

A comparação mostra ainda que, enquanto para a via linear o avanço do passo de rendimento reduzido provoca um aumento monótono dos custos, no caso da via convergente ocorre uma queda brusca do aumento de custos quando se passa da posição 5 para a 6 . Este facto resulta de, nesta via, haver duas linhas que convergem para realizar a última reação - a queda brusca corresponde à mudança da primeira linha para a segunda. Em cada linha, os custos aumentam com a progressão do passo de rendimento reduzido, o que está de acordo com o facto de se dever colocar um passo de baixo rendimento preferencialmente no início da via, para minimizar os custos. Os custos para rendimentos reduzidos dos passos 2 e 3 são os mesmos, porque estes podem ser realizados por esta ordem ou pela inversa (a estrutura da via convergente é simétrica quanto à formação dos intermediários $\mathrm{AB}$ e CD). Uma comparação em globo mostra ainda que o abaixamento de rendimento num passo da linha secundária $(6 \rightarrow 7 \rightarrow 8 \rightarrow 9)$ tem menor influência nos custos do que se ocorrer na principal ( $1 \rightarrow 2 \rightarrow 3 \rightarrow 4 \rightarrow 5$ ), o que evidencia o aumento de robustez conseguida com a convergência.

A Figura 5 mostra como variam a economia atómica e o factor E com o deslocamento do passo de baixo rendimento. Quando se compara esta figura com a referente à via linear, notam-se diferenças do mesmo tipo que para o caso dos custos (a Figura 5 foi desenhada à mesma escala da Figura 2 da ref. 1 para facilitar a comparação, não sendo essa escala a mais adequada para a representação apresentada aqui). Por um lado, as variações dos parâmetros de produtividade atómica são mais reduzidas para o caso da via convergente, sendo sempre a economia atómica superior, e o fator E inferior - ou seja, a via convergente apresenta maior produtividade atómica que a via linear perante a redução do rendimento de um passo. Por outro lado, ocorrem flutuações, não variações monótonas, por estarem em jogo movimentos ao longo de duas linhas convergentes - mas para cada uma destas ocorrem variações monótonas, mostrando que para a maximização da produtividade atómica o passo de baixo rendimento deve ser colocada no início das linhas. Em suma, a via convergente é mais robusta também quanto à verdura atómica.

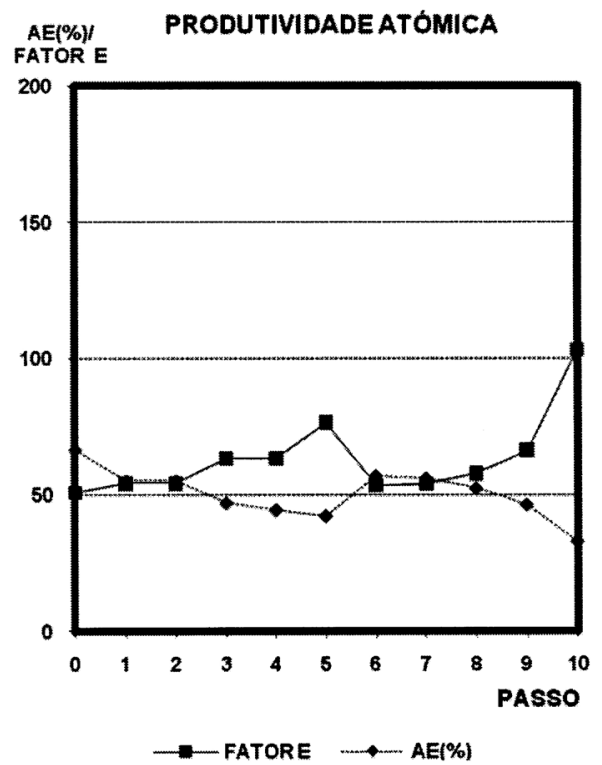

Figura 5. Variação da economia atómica e fator $E$ da via de síntese convergente em função da posição do passo com rendimento $50 \%$

Finalmente, a análise conjunta das Figuras 4 e 5 comprova, como na ref. 1 , que a perseguição do aumento da verdura, traduzida aqui pela produtividade atómica, proporciona paralelamente economia de custos - ou seja, que a QV pode funcionar como fator de mais-valia económica da química.

\section{OUTRAS COMPARAÇÕES}

A seguir analisam-se brevemente os outros aspetos de comparação das vias linear e convergente listadas no Quadro 2, com vista a avaliar os seus efeitos na verdura relativa.

\section{Menos trechos de via improdutivos}

Os diferentes trajetos dos diversos conjuntos de átomos que são integrados como fragmentos no produto final nas duas vias de síntese implicam trechos improdutivos mais longos na via linear estes trechos são constituídos pelas partes dos percursos em que um dado fragmento não participa em reações, mas apenas é transportado entre reações da via em que intervém. Como se sabe, os bilhetes de transporte custam sempre dinheiro, que paga a energia para a movimentação e o investimento nos veículos - e o caso das "viagens" de fragmentos intactos nas vias de síntese não é excepção: também aqui o transporte custa sempre energia (a comparação não é perfeita, porque no caso das vias de síntese não há passagem grátis!) e implica maiores gastos nos "veículos de transporte", já que o processamento 
de maior massa requer peças de equipamento de maior volume e, portanto, mais caras. Também quanto a este aspeto a via convergente potencia a verdura, já que poupa energia (e não só).

\section{Controlo de impurezas}

Nos trajetos improdutivos em termos de transformação progressiva das moléculas de substrato para o produto final, os fragmentos que não participam nela ficam expostos a reações laterais indesejadas, que se traduzem num consumo virtual de uma parte do produto final - e, eventualmente, a sua contaminação por impurezas (usando a metáfora anterior da viagem, às vezes o turista tem de fazer trechos de percurso que não lhe interessam, só para chegar aos locais que quer visitar - esses trechos, até porque são desinteressantes, maçam o viajante!). Por isso, a seletividade obtida com vias convergentes é geralmente superior à das lineares - já que os fragmentos são menos sujeitos a sucessivas condições de reação diferentes que possam fazêlos participar em reações laterais.

Ainda quanto às impurezas no produto final, a síntese convergente, além de permitir diminuir a sua variedade e quantidades pelo efeito anterior, tem outra vantagem. Se a síntese convergir só no fim, ocorre aí um aumento brusco da complexidade (e da massa) molecular ao formar-se o produto final, pelo que as impurezas serão, em geral, menos semelhantes a este - e, portanto, em princípio, mais fáceis de separar. Em contraste, numa via linear, o aumento de complexidade ocorre em pequenos incrementos ao longo de todo o percurso, havendo possibilidades de a complexidade das impurezas ir aumentando paralelamente com a elaboração química da molécula, realizada progressivamente até se chegar ao produto. Usando como exemplo as vias na Figura 1, o último intermediário da via linear (ABCDEFGHIJ) é mais semelhante ao produto (ABCDEFGHIJK) do que na via convergente, em que se faz a montagem da molécula final a partir dos segmentos (ABCDEF) e (GHIJK), pelo que a eliminação do produto dos reagentes remanescentes será mais difícil no primeiro caso. A minimização da necessidade de separações para purificação significa aumento de verdura.

\section{Aquisição exterior de intermediários}

O processamento paralelo operado nas sínteses convergentes permite que se possam usar mais facilmente intermediários adquiridos no mercado, ou mandados fazer por encomenda (custom synthesis), o que pode ter interesse para acelerar a produção, se isso for economicamente vantajoso. Este aspeto pode ter influência na verdura, mas os efeitos variam de caso para caso, tendo de ser analisados em cada situação concreta.

\section{Discussão}

De modo geral, as vias de síntese convergentes comparam-se favoravelmente com as lineares: permitem o fabrico de compostos com menores custos, quer de reagentes estequiométricos e auxiliares (solventes, etc.), quer de conversão (menos energia, equipamentos menos volumosos, etc.); possibilitam um melhor controlo das impurezas no produto; fornecem maior versatilidade quanto ao aprovisionamento/ fabrico de compostos intermediários, etc. Por ação de muitos destes fatores, as vias convergentes suportam a maximização da verdura, em especial a verdura atómica aqui analisada.

\section{CONCLUSÕES FINAIS}

O cálculo do rendimento de uma via de síntese por multiplicação dos rendimentos dos passos da linha mais longa pode ter um significado problemático - um valor maior de rendimento, por si só, não implica uma eficiência elevada da via. As limitações do rendimento global são particularmente agudas no caso das vias convergentes e comparações com base nesta métrica devem ser analisadas com muito cuidado.

As vias convergentes têm vantagens logísticas sobre as correspondentes vias lineares, porque implicam menor extensão de transporte de fragmentos intactos entre reações ao longo da via e são mais robustas quanto a perturbações nas condições de realização. Além disso, apresentam maior seletividade e menor necessidade de grupos protetores e, graças à convergência de linhas que permite a elaboração paralela de fragmentos, possibilitam a redução do tempo de realização do ciclo de fabrico - permitem maiores velocidades de fabrico. $\mathrm{O}$ aumento abrupto da complexidade (e massa) molecular nos pontos de convergência representa uma vantagem em termos de diferenciação e separação de impurezas. Finalmente, a versatilidade quanto à aquisição exterior de intermediários é maior nas vias convergentes. Muitas destas vantagens implicam também um aumento da verdura da síntese.

Em conclusão, quando se procura obter otimizar a síntese de um composto para fabrico industrial, deve-se ter sempre em mente que vale a pena considerar rotas convergentes para chegar a uma via de síntese eficaz e tão verde quanto possível - que satisfaça na maior extensão possível as características óptimas da chamada via de síntese verde. ${ }^{17}$

Finalmente, este estudo mostra, mais incisivamente que o anterior, ${ }^{1}$ como é importante que os químicos adquiram uma mentalidade sistémica para lidarem com a perseguição da verdura preconizada pela QV. Esta tarefa compreende um grande número e variedade de fatores a considerar simultaneamente, não só individualmente mas também com respeito às suas interrelações e ao seu todo, para atingir o objetivo final de obter um processo de manufatura do composto à escala industrial economicamente exequível e com verdura máxima - este objetivo é multidimensional, pelo que o problema requer pensamento holístico e práticas sistémicas.

\section{REFERÊNCIAS}

1. Machado, A. A. S. C.; Quim. Nova 2011, no prelo.

2. Zhang, T. Y. Em Handbook of Green Chemistry and Technology; Clark, J.; Macquarrie, D., eds.; Blackwell: Oxford, 2002, p. 306-320.

3. Walker, D.; The Management of Chemical Process Development in the Pharmaceutical Industry, Wiley-Interscience: Nova Iorque, 2008.

4. Anderson, N. G.; Practical Process Research \& Development, Academic Press: San Diego, 2000.

5. Zhang, T. Y.; Chem. Rev. 2006, 106, 2583.

6. Fuchs, P. L.; Tetrahedron 2001, 57, 6855.

7. Hudlicky, T.; Chem. Rev. 1996, 96, 3.

8. Steinbach, A.; Winkerbach, R.; Chem. Eng. 2000, april, 94.

9. Trost, B. M.; Science 1991, 254, 1471.

10. Sheldon, R. A.; Chem. Ind. (London) 1992, 903.

11. Machado, A. A. S. C.; Química-Bol. S. P. Q. 2007, 107, 47.

12. Anastas, P. T.; Warner, J. C.; Green Chemistry - Theory and Practice, Oxford UP: Oxford, 1998, p. 30.

13. Lenardão, E. J.; Freitag, R. A.; Dabdoub, M. J.; Batista, A. C. F.; Silveira, C. C.; Quim. Nova 2003, 26, 123.

14. Machado, A. A. S. C.; Química-Bol. S. P. Q. 2004, 95, 59.

15. Esta atitude é preconizada com generalidade pela sistémica para a simplificação dos sistemas: antes de realizar simplificações para a obtenção de modelos manejáveis é essencial compreender bem o sistema - para que o modelo simplificado não perca caraterísticas do sistema que é essencial considerar para o fim em vista (ver, p. ex., de Bono, E.; Simplicity, Penguin: Londres, 1999).

16. Machado, A. A. S. C.; Química-Bol. S. P. Q. 2010, 118, 41.

17. Machado, A. A. S. C.; Química-Bol. S. P. Q. 2011, 120, 43. 